\title{
Influence of surface recrystallization on the low cycle fatigue behaviour of a single crystal superalloy
}

\author{
X. MA ${ }^{1,2}$, H.-J. SHI ${ }^{1}$, J. GU ${ }^{3}$, Z. YANG ${ }^{1}$, G. CHEN ${ }^{4}$, O. LUESEBRINK ${ }^{5}$ and H. HARDERS ${ }^{5}$ \\ ${ }^{1}$ AML, School of Aerospace, Tsinghua University, Beijing 100084, China, ${ }^{2}$ Department of Materials Science and Engineering, University of Michigan, \\ Ann Arbor, MI 48109, USA, ${ }^{3}$ Department of Material Science and Engineering, Tsinghua University, Beijing, China, ${ }^{4}$ Siemens Corporate Technology, \\ Beijing, China, ${ }^{5}$ Siemens AG Energy, Mülheim an der Rubr, Germany
}

Received Date: 17 January 2014; Accepted Date: 13 fuly 2014; Published Online: 19 September 2014

\begin{abstract}
This paper investigated the effect of surface recrystallization (RX) on the low cycle fatigue (LCF) behaviour of a single crystal (SX) superalloy. LCF tests on both raw and recrystallized samples showed that fatigue life was significantly reduced by surface $\mathrm{RX}$. Fractography indicated that fatigue cracks initiated from the casting defects in RX layer and multiple crack initiations were commonly observed. Moreover, RX grains exhibited predominantly transgranular cracking, in contrast to the intergranular fracture reported in literature. The fatigue crack propagation behaviour was discussed in light of fracture mechanics and crack growth life model. The fatigue cycles required to penetrate RX layer were estimated to be about one magnitude lower than that in forming an equivalent crack in SX specimens. It is suggested that the earlier crack initiation and promoted crack propagation in RX layer, as well as the trend of multiple initiations, are responsible for the fatigue degradation by RX.
\end{abstract}

Keywords crack initiation; crack propagation; low cycle fatigue; recrystallization; single crystal superalloy.

N O M E N C L A T URE $\quad \begin{aligned} a & =\text { crack length or depth } \\ a_{0} & =\text { initial crack size } \\ a_{f} & =\text { final crack size before fracture } \\ C, n & =\text { Paris parameters } \\ C_{1}, m & =\text { model parameters } \\ d a / d N & =\text { crack growth rate } \\ E_{R} & =\text { Reuss's average modulus } \\ N_{f} & =\text { number of cycles to failure } \\ N_{p} & =\text { fatigue crack growth life } \\ N_{i} & =\text { fatigue crack initiation life } \\ N_{p \mathrm{RP}} & =\text { fatigue crack growth life in recrystallization layer } \\ N_{f \mathrm{RX}} & =\text { fatigue life cycles consumed in recrystallization layer } \\ N_{f \mathrm{SX}} & =\text { fatigue crack growth life in single crystal substrate } \\ S_{11}, S_{12}, S_{44} & =\text { elastic compliance constants } \\ \Delta K & =\text { stress intensity factor range } \\ \sigma_{a} & =\text { stress amplitude }\end{aligned}$

\section{INTRODUCTION}

Recrystallization (RX) is a well-known issue in the casting processing and service of turbine blades made of directionally solidified (DS) and single crystal (SX)

Correspondence: H.-J. Shi. E-mail: shihj@mail.tsinghua.edu.cn nickel-based superalloys. ${ }^{1,2}$ Plastic deformation might be introduced by several possible sources during manufacturing and processing of the new blades as well as during service and reconditioning. ${ }^{1,3,4}$ And $\mathrm{RX}$ would occur if the deformed parts were exposed to a high enough temperature in any of the processes. In recent decades, the RX problem has gained notable 
increasing attention from both scientists and engineers, because of the demanding safety requirements of modern turbine components used in aeronautic engine and industrial land base gas turbine. ${ }^{1-3,5-8}$ It has been accepted that surface RX of nickel-based superalloys may be responsible for the performance deterioration and failure of DS and SX turbine blades. ${ }^{1,2}$ And it is proposed $^{2,8}$ that the transverse grain boundaries introduced by recrystallized grains can act as the preferred crack initiation site due to lack of grain boundary strengthening elements (e.g. B, Hf and $\mathrm{Zr}$ ) in SX superalloys. ${ }^{3}$

While there are extensive studies focusing on the influence factors ${ }^{5,7}$ and microstructural morphologies $^{5,7,9,10}$ of RX, degradation of tensile ${ }^{11}$ and creep properties ${ }^{6,11-13}$ by RX, less attention has been paid to the influence of RX on low cycle fatigue (LCF) behaviours. ${ }^{3,8}$ Among the limited research in open literature, studies on different alloy systems under different fatigue test conditions have not yet produced conclusive results. For instance, it is reported that remarkable decrease in fatigue life can be observed in CMSX- $4,{ }^{3} \mathrm{DZ} 4^{2,14}$ and $\mathrm{DZ} 40 \mathrm{M}^{8}$ superalloys with surface RX. However, the work of Bürgel et al. ${ }^{1}$ on CMSX-6 superalloy with $0.1 \mathrm{~mm}$ surface recrystallized layer revealed no significant difference in crack initiation life between the RX and raw samples, except for a much higher crack density in $\mathrm{RX}$ layer. Moreover, it is recently found in DZ4 DS superalloy $^{15}$ that the sample with RX layer generated under a high-pressure shot peening exhibited even higher fatigue life than that of raw DZ4 superalloy. It is demonstrated that the discrete $\mathrm{RX}$ grains are most harmful to the fatigue properties, whereas compact

Table 1 The chemical composition of single crystal superalloy (wt pct)

\begin{tabular}{lcccccccc}
\hline $\mathrm{C}$ & $\mathrm{W}$ & $\mathrm{Cr}$ & $\mathrm{Co}$ & $\mathrm{Mo}$ & $\mathrm{Ti}$ & $\mathrm{Al}$ & $\mathrm{Ta}$ & $\mathrm{Ni}$ \\
\hline 0.067 & 3.9 & 12.0 & 9.0 & 2.0 & 4.0 & 3.6 & 5.0 & Balanced \\
\hline
\end{tabular}

recrystallized layer does not necessarily lead to degradation in fatigue life. ${ }^{15}$ Besides, there is another issue, that is, whether the recrystallized grain boundaries are always the preferential crack initiation site, or if not, the critical influence factors have been rarely clarified to the author's knowledge. Hence, further experimental investigation is in great need to address and add lights to these questions.

In the present study, a $150 \mu \mathrm{m}$ uniform RX layer with one grain through the depth was prepared on a SX superalloy by shot peening and subsequent heat treatment. Further, a series of LCF tests were performed at $550^{\circ} \mathrm{C}$, for specimens with and without RX. The effects of continuous RX layer on the LCF behaviour of SX superalloy were extensively studied, together with fractography and microstructural observations, to understand the characteristics of fatigue crack initiation and propagation. An evaluation of fatigue crack growth life provided insight into the life percentage consumed in RX layer and helped to understand its influence on fatigue life. This study also aimed to provide a more comprehensive viewpoint in understanding the fracture mode and life degradation of RX layer and hence benefits the maintenance of SX components with respect to RX issue.

\section{MATERIAL AND EXPERIMENTAL PROCEDURE}

The material used in this study is a SX nickel-based superalloy with a nominal composition as shown in Table 1. The high content of $\mathrm{Cr}$ is to promote its hot corrosion resistance. SX rods were DS to produce a [001] crystal orientation. The crystallographic orientation along the axis of the bar was determined to be within $5^{\circ}$ of dispersion using the Laue $\mathrm{X}$-ray diffraction technique as described in Ref. [16] The as-received superalloy has a two-phase microstructure (Fig. 1), which consists of a face-centred cubic $\gamma$ phase and a precipitated $\mathrm{L}_{2} \gamma^{\prime}$ phase. Some blocky carbides rich in Ta are widely distributed, ${ }^{17}$
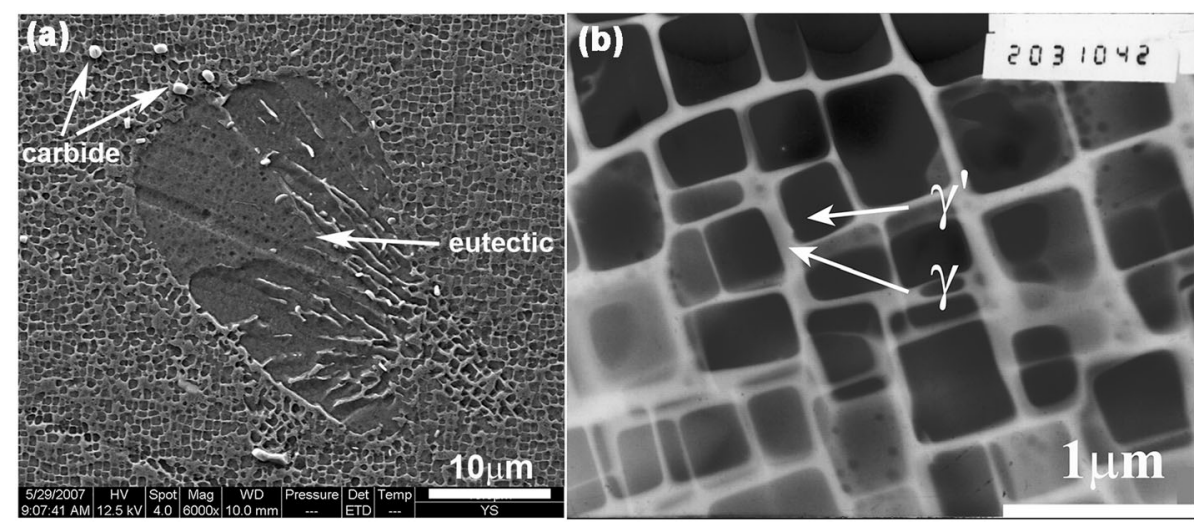

Fig. 1 Typical microstructure of the single crystal superalloy (a) scanning electron microscope micrograph (b) transmission electron microscopy micrograph. 
and eutectic pools can be observed occasionally in metallographic examinations (Fig. 1a). Figure 1b is a typical transmission electron microscope image of this SX superalloy, in which the $\gamma^{\prime}$ precipitates have an average size of $0.5 \mu \mathrm{m}$.

The dimensions of LCF testing specimen are shown in Fig. 2a. Figure 2b shows an example of the as-machined fatigue specimen. All LCF specimens have the longitudinal axis oriented along the nominal [001] direction. The SX bars were subjected to shot peening at $0.45 \mathrm{MPa}$ for $10 \mathrm{~min}$ (about $2 \times 98 \%$ coverage) to produce a uniform deformation layer on the sample surface. (Fig. 2c). The deformed samples were then sealed in a quartz tube in which a positive pressure of argon gas was maintained. A solution and precipitate heat treatment was performed followed by air cooling, to be consistent with the standard procedure of turbine blade in practice. The LCF specimens with recrystallized layer were polished using $3 \mu \mathrm{m}$ and $1 \mu \mathrm{m}$ diamond paste prior to LCF testing (Fig. 2d).

The LCF tests of both raw SX specimens and recrystallized specimens were conducted in ambient atmosphere under fully reversed $(\mathrm{R}=-1)$ axial strain control mode, employing a SHIMAZU servo-hydraulic system of $\pm 98 \mathrm{kN}$ dynamic load capacity. Axial strain was measured and controlled from an axial gauge length extensometer mounted on the test specimen prior to LCF testing. A strain rate of $10^{-3} \mathrm{~s}^{-1}$ was employed for all tests. The specimens were heated in a furnace installed on the fatigue machine. Uniform heating of the specimen gauge length was attained by carefully monitoring and controlling the specimen temperature using three separately adjustable thermocouples. The temperature profile remained within about $\pm 2{ }^{\circ} \mathrm{C}$ of the nominal temperature throughout each test. To understand the crack initiation and propagation of recrystallized samples, seven fatigue specimens with $150 \mu \mathrm{m} \mathrm{RX}$ were subjected to interrupted tests under the same strain amplitude. The drop-in load was measured by decrease of force compared with the stabilized force or the force at middle life. Fractography of all fatigue specimens and microstructural analysis were performed using a scanning electron microscope (SEM) of Quanta $200 \mathrm{~F}$.

\section{RESULTS AND DISCUSSION}

\section{Recrystallization microstructure}

Figure 3 shows the typical microstructure of RX on the fatigue specimen surface. The RX depth was about
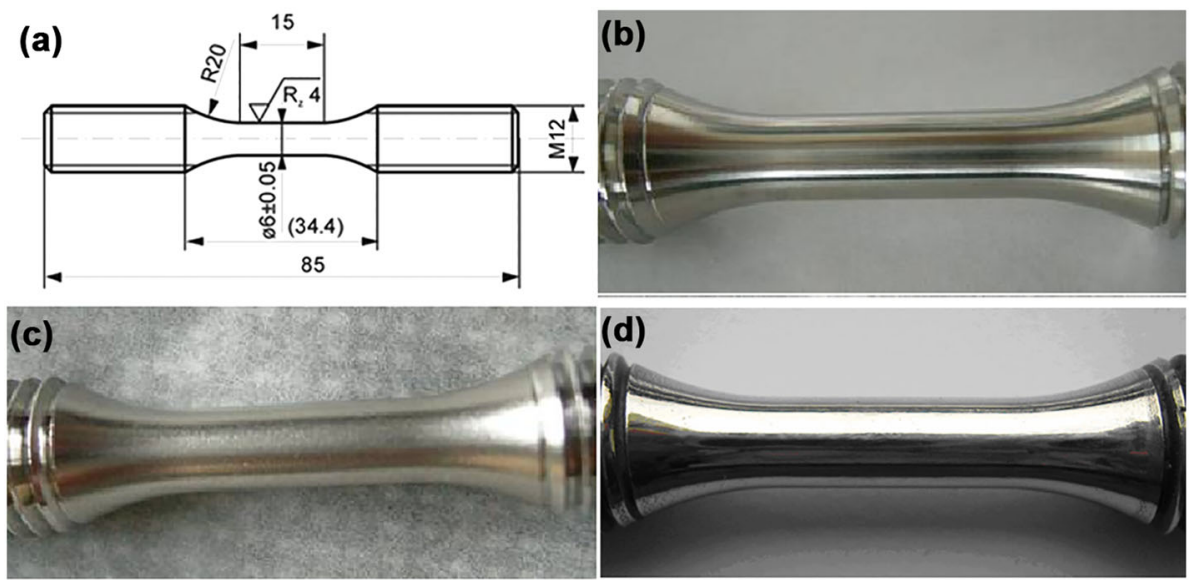

Fig. 2 Low cycle fatigue testing specimen: (a) specimen dimension (all dimensions in mm), (b) as-machined, (c) after shot peening and (d) after recrystallization and polishing.

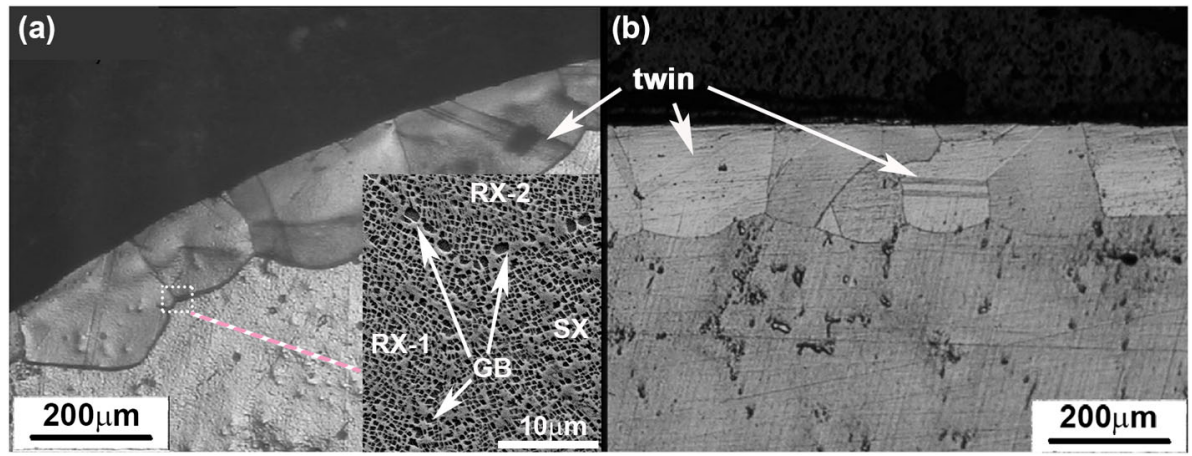

Fig. 3 Optical microstructure of surface recrystallized specimen: (a) transverse section and (b) longitudinal section. 
$150 \mu \mathrm{m}$ on average in Fig. 3a and b. Of interest is that there is generally a single grain through the RX layer thickness in all samples, which is similar to that obtained in CMSX-2 superalloy. ${ }^{12}$ Residual stresses originated from shot peening assisted dissolution of the existing phases during the solution heat treatment. Annealing twins can be observed in some RX grains, as arrowed in Fig. 3a and b.

The inset in Fig. 3a is a SEM micrograph of RX grains showing the triple joints. The RX grains have comparable microstructure with the substrate SX superalloy, in terms of the size and morphology of $\gamma^{\prime}$ precipitates. This is distinct from the cellular RX microstructures with coarse $\gamma^{\prime}$ obtained in Bürgel's ${ }^{1}$ and Okazaki's work. ${ }^{3}$ It is reasonable as the present study used a solution heat treatment above the $\gamma^{\prime}$ solvus, where equiaxed $\mathrm{RX}$ grains containing comparable $\gamma^{\prime}$ size were expected because of the absence of most of the barriers, for example, $\gamma^{\prime}$ particles and eutectics. ${ }^{5}$ The RX grain boundaries contain a higher fraction and slightly larger size of $\gamma^{\prime}$ precipitates, which can be evidently observed in Fig. 3a.

\section{Strain-life curve}

The strain amplitude was plotted in terms of the number of cycles to failure for specimens with and without RX, shown in Fig. 4. It is seen that the $150 \mu \mathrm{m}$ surface RX layer has a significant effect on the LCF life of SX superalloy. Taking the specimens under the same amplitude (i.e. $\varepsilon_{3}$ ), for example, the average fatigue life of raw specimens is around 11400 cycles, whereas that of RX specimens is 1347 cycles. That is to say, the LCF life was about one magnitude lower because of RX. With higher strain amplitude (i.e. $\varepsilon_{2}$ ), the fatigue life was also reduced by about $80 \%$. The results in Fig. 4 indicated that the RX had remarkable detrimental influence on

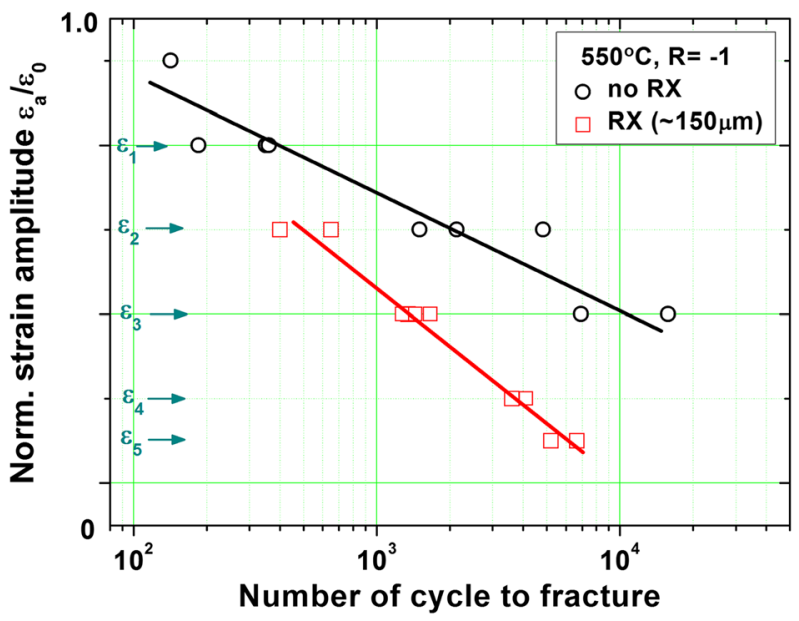

Fig. 4 Low cycle fatigue strain-life curves of raw single crystal and recrystallized specimens. the LCF life of SX superalloy. This degradation of fatigue life by partial RX is similar to that reported in other SX superalloy ${ }^{3}$ and DS superalloys, ${ }^{8,18}$ despite of different alloy systems and testing conditions.

\section{Fatigue fracture behaviour Fractography}

To understand the observed effect of surface RX layer on fatigue life, all samples were examined under SEM after fatigue tests. For raw SX specimens, the overall appearance of the fracture surfaces at $550^{\circ} \mathrm{C}$ revealed that fatigue fracture occurred predominately on either one (Fig. 5a) or multiple crystallographic planes (Fig. 6a). Higher magnification of the crack initiation sites (region 'b' in Fig. 5a) showed that casting pores or defects are the primary crack initiators in this superalloy (arrowed in Figs $5 \mathrm{~b}$ and $6 \mathrm{~b}$ ). The aforementioned observation agrees with our former studies of this superalloy at intermediate temperature. ${ }^{17}$ The crack propagation was inclined about $50^{\circ}$ from the specimen axis (i.e. [001]), indicating that the crack propagated on an octahedral slip plane, which is the preferred cracking mode for SX superalloy at low temperatures or high frequencies $(>3 \mathrm{~Hz}) .{ }^{17}$ Figure $5 \mathrm{c}$ is a higher magnification observation of region ' $c$ ' in Fig. 5a, where river patterns were readily observed. Figure $5 \mathrm{~d}$ shows the fracture at high $\Delta \mathrm{K}$ region before final rupture, where ridges were formed by fracture on two intersecting $\{111\}$ planes.

For recrystallized specimens, it is found that fatigue fracture was initiated from the surface RX layer, where casting pores (Figs 7c and 8a) or other defects (Figs 7b and $8 \mathrm{~b}$ ) acted as the fatigue crack initiators. It should be noted that the RX grains exhibited transgranular cracking mode, that is, fatigue cracks passed through RX grains, indicated by the crystallographic fracture features in Figs $7 \mathrm{~b}-\mathrm{d}$ and 8 . This is distinct from the widely reported intergranular cracking of recrystallized grains under either fatigue ${ }^{3,8}$ or creep $^{11,12}$ loading conditions in previous studies. According to the fractography of all fatigue samples, recrystallized grain boundaries were not the preferred crack sites in this study. The underlying mechanism will be discussed in the following sections. After penetrating the RX layer, the crack propagation in the substrate SX superalloy occurred via crystallographic cracking, that is along $\{111\}$ slip planes (marked in Figs $7 \mathrm{~b}$ and 8), similar to those in Figs 5a and 6b. River pattern features were evident on the $\{111\}$ planes.

Besides, some RX grains can be identified on the fracture surfaces (Figs 7b-d and 8a and b), according to the different appearance of fracture features, which is mainly due to the different crystal orientations of RX grains against the SX substrate. Also, it is emphasized that recrystallized specimens preferred multiple fatigue crack 


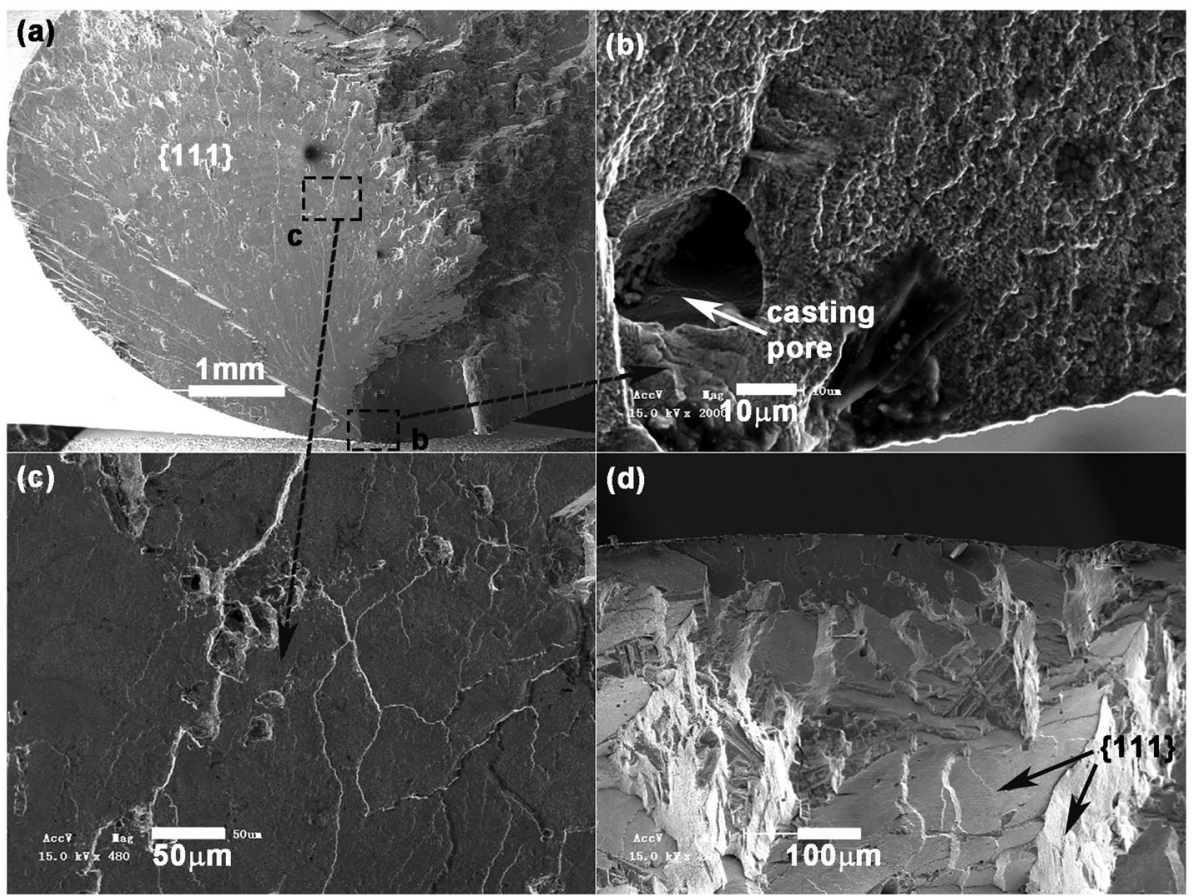

Fig. 5 Fatigue fracture surface of raw single crystal specimen (6919 cycles): (a) overview of fracture surface, (b) crack initiation from casting pore, (c) cleavage fracture and (d) ridges in high $\Delta \mathrm{K}$ region.

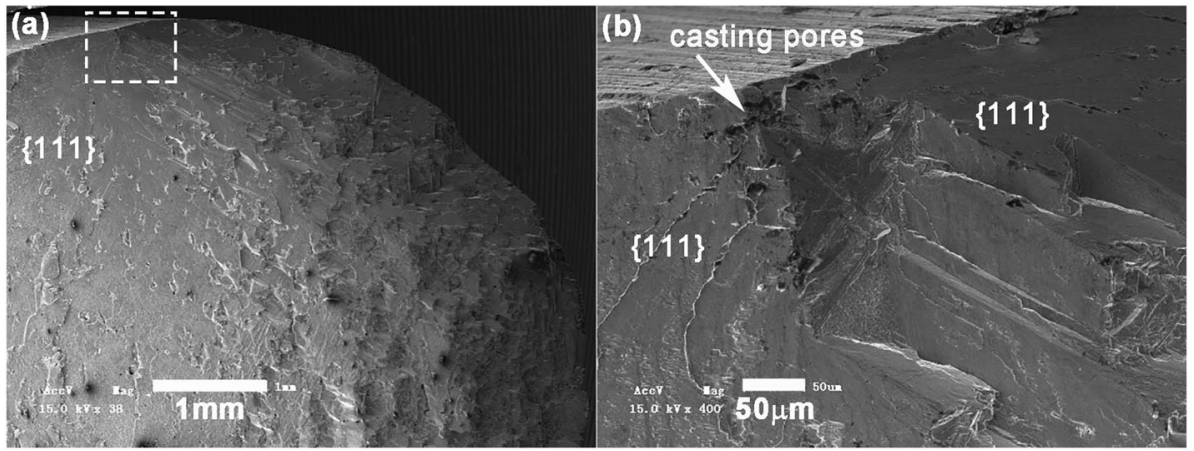

Fig. 6 Fatigue fracture surface of raw single crystal specimen (185 cycles): (a) overview of fracture surface and (b) crack initiation from casting pores.

initiation, as shown in Figs 7c and 8a. This feature was commonly observed in all RX samples. Hence, it is indicated that the degradation effect of RX layer is partly due to the increased possibilities of fatigue crack initiation from multiple sites.

\section{Sectioned microstructure analysis}

Some fatigue specimens were sectioned near the crack initiation site to disclose the fracture features beneath the surface. The longitudinal section of an SX specimen is shown in Fig. 9, which reveals a casting micropore and a blocky carbide at the crack initiation site. An energy dispersive spectroscopy analysis indicated that the carbide is rich in Ta, which is a secondary crack initiator in the studied superalloy. ${ }^{17}$ It is reported that Ta-rich blocky carbides would act as the primary crack initiators in this series of SX superalloys after reducing the casting porosity by advanced hot isostatic pressing techniques. ${ }^{19}$

For recrystallized specimens, the fatigue cracking features on longitudinal section were shown in Fig. 10. The RX grain boundary was observed beneath the initiation site (arrowed) in Fig. 10a, indicating a transgranular fracture mode of the primary crack, which coincides with the fractography depicted earlier. Some secondary transgranular cracks were also found, for instance, in the RX grains in Fig. 10b. Besides, intergranular cracking was occasionally observed in the examined samples, see Fig. 10c, where another transgranular crack (arrowed) was also observed. Figure 10d shows a crack initiated at the RX grain boundary, which evolved into transgranular mode eventually. The observations in the preceding text 

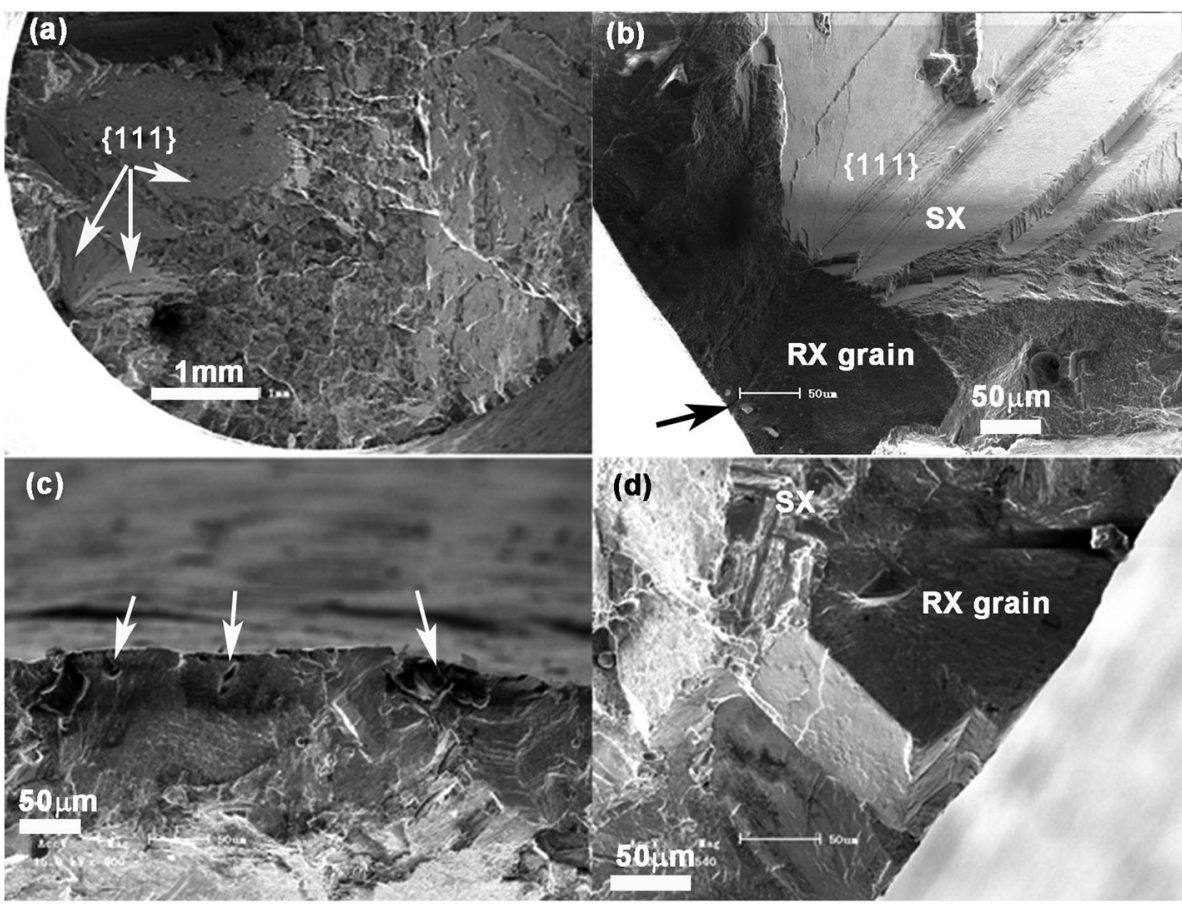

Fig. 7 Fatigue fracture of specimen with $150 \mu \mathrm{m}$ recrystallization layer (658 cycles): (a) overview of fracture surface, (b) primary initiation site, (c) multiple initiations from casting defects and (d) crystallographic fracture.

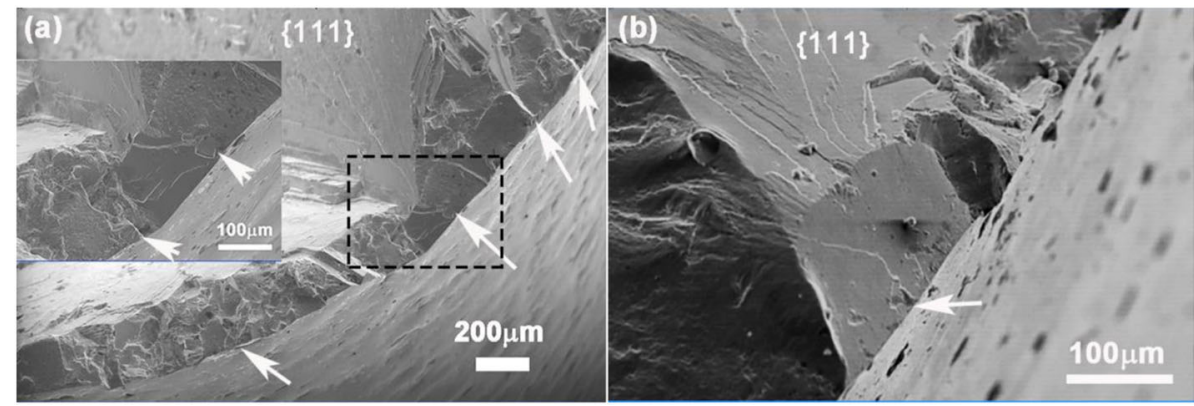

Fig. 8 Fatigue fracture of specimens with $150 \mu \mathrm{m}$ recrystallization layers: (a) primary and secondary crack initiation sites (478 cycles) and (b) primary crack initiation site (6688 cycles).

indicate that transgranular cracking is the predominant fracture mode of RX grains in the SX superalloy under study. It reminds one about the temperature-dependent strength of intra-grain and grain boundary. At temperatures below the so-called iso-strength temperature $T_{s}$, transgranular cracking would be favoured; whereas intergranular cracking would be dominant above $T_{s}$. The author's recent studies on DZ4 superalloy ${ }^{15,20}$ have also suggested that the difference in fracture mode is attributable to test condition, especially the temperature, that is, transgranular cracking dominates below $T_{\mathrm{s}}$ (between 350 and $760^{\circ} \mathrm{C}$ for DZ4 alloy ${ }^{15}$ ). For the SX superalloy under study, it is known that it prefers crystallographic shearing fracture mode at $600{ }^{\circ} \mathrm{C}$ or below; $;^{17}$ thus, the transgranular cracking observed in $\mathrm{RX}$ grains can be somewhat rationalized. Intergranular cracking of recrystallized layer can be expected at much higher temperatures, which is being studied by additional tests and will be shown in a follow-up work. ${ }^{21}$

Despite the aforementioned observations of transgranular cracking, some other features in the sectioned microstructure can be identified. In Fig. 11a, a crack initiated from a coarse eutectic in the RX grain. Also it is observed that the fatigue crack has an evident interaction with local microstructural inhomogeneity. In Fig. 11b, the crack passed through the carbide, penetrated the RX/SX interface, coalesced with the casting pore and continued to propagate. Similarly, in Fig. 11c, the crack penetrated the carbides in the way. The transgranular crack in Fig. 11d was arrested by the carbide ahead. From this point of view, the carbides can act as barriers of fatigue crack propagation, although it can be a potential crack 

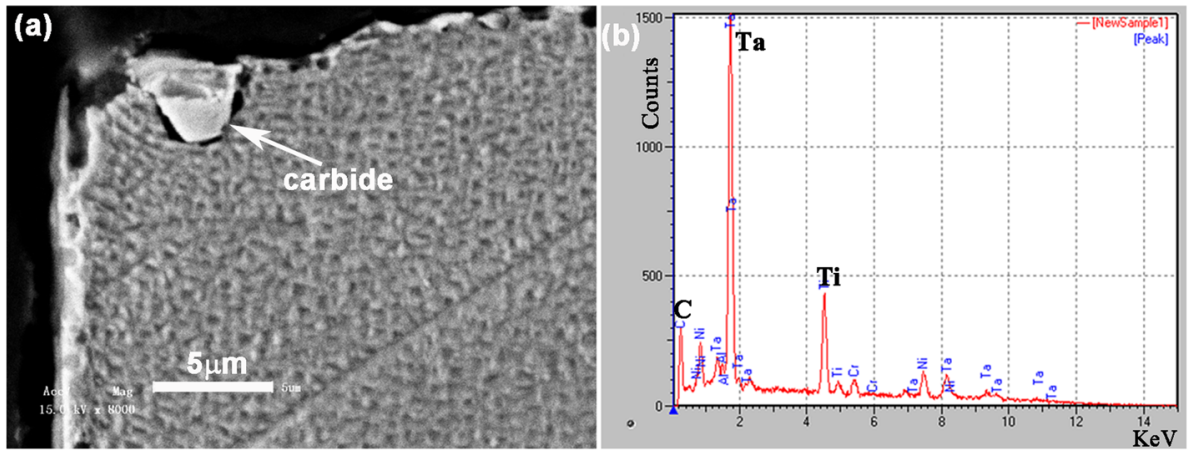

Fig. 9 Sectioned microstructure of the raw single crystal superalloy: (a) crack initiation site and (b) energy dispersive spectroscopy result of the carbide.
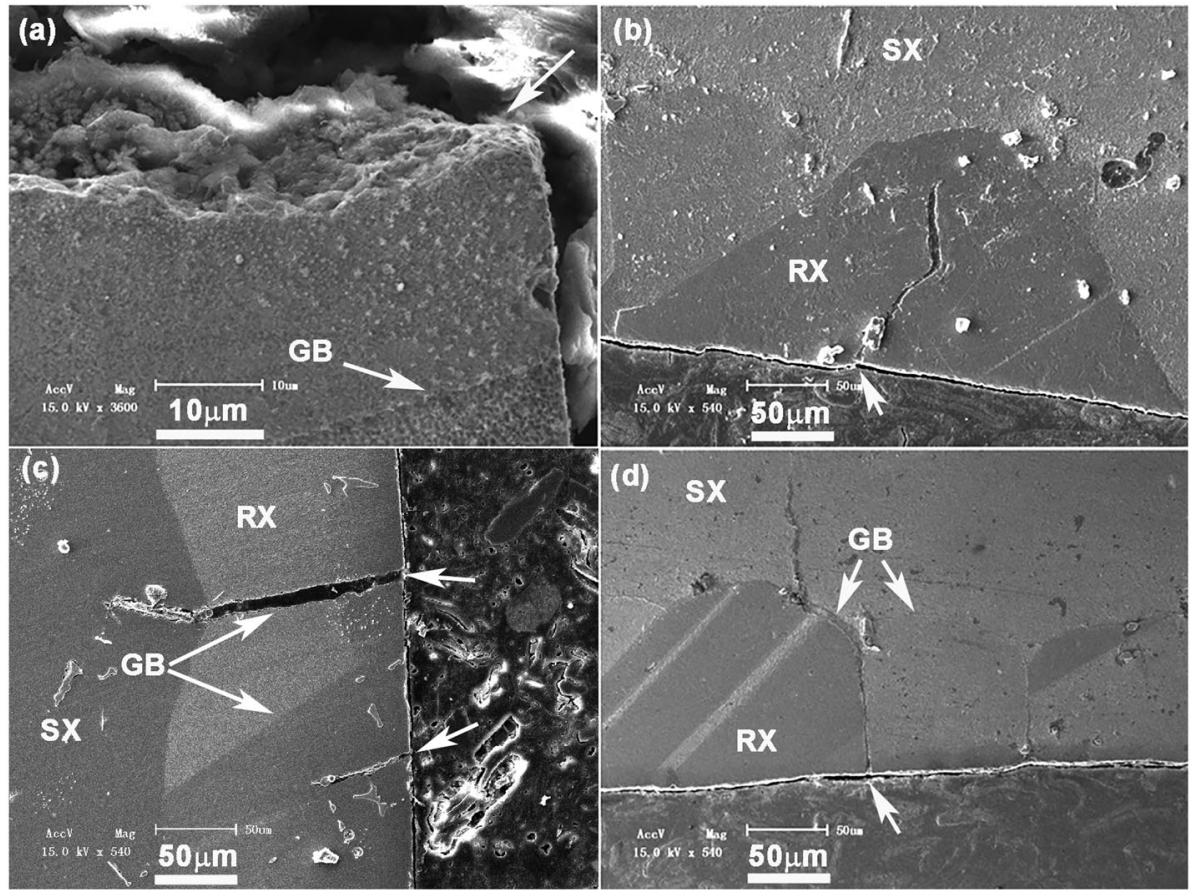

Fig. 10 Fatigue cracking behaviour on the sectioned $150 \mu \mathrm{m}$ RX samples: (a) transgranular cracking at initiation site, (b) secondary transgranular crack, (c) coexistance of intergranular and transgranular cracks and (d) transition from intergranular to transgranular cracking.

initiator in some case. The observations in this study revealed the primary microstructural inhomogeneities that are responsible for crack initiation, including casting pores (Figs 7c, 8a and 11d), eutectic (Fig. 11a) and carbides (Fig. 11c). These surface or near-surface defects could lead to stress concentration and localized plastic deformation to facilitate fatigue crack initiation.

\section{Fatigue crack propagation behaviour}

Some fatigue tests of RX samples under constant strain amplitude were interrupted to obtain more information about crack propagation. These samples were expected to have a target fatigue life of about $1300 \sim 1400$ cycles.
The LCF tests were interrupted at different load drop values, that is, $0 \%, 3 \%, 5 \%, 8 \%$, to study the crack evolution with damage. It is seen in Fig. 12 that the drop-in load has no evident relationship with the fracture of fatigue specimen. In sorting the fatigue cycles of each specimen, it is found that the RX specimens would survive before 1218 cycles and break after 1274 cycles.

A non-destructive inspection technique, that is, fluorescence penetrant flaw detection, was used to detect the surface cracks in the interrupted specimens (Fig. 13a). The cracks were marked, and then the specimens were sectioned to examine the depth and path of fatigue cracks. It is seen in Fig. 13b that after 882 cycles of fatigue loading, the crack has already penetrated through 


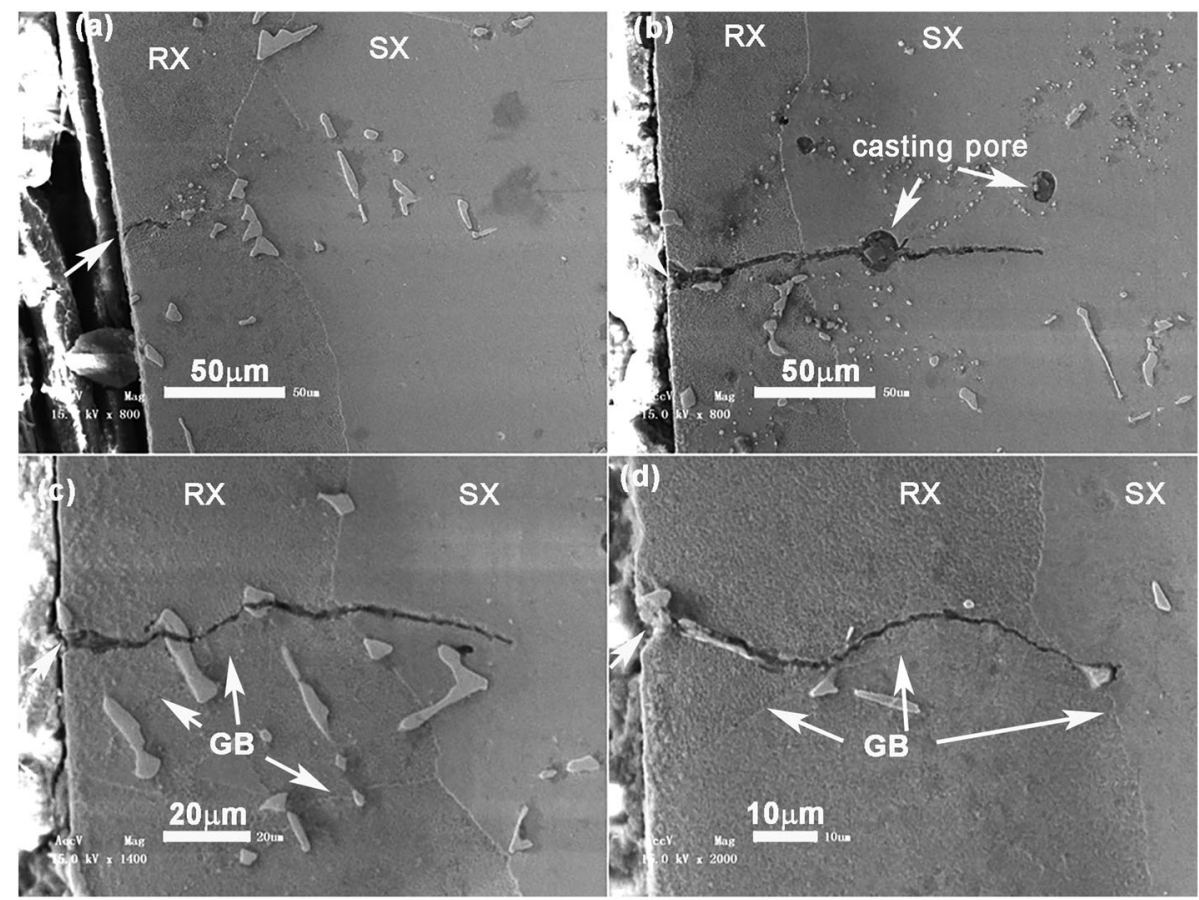

Fig. 11 Fatigue cracking features on longitudinal section: (a) fatigue crack originated from eutectic, (b) transgranular crack passed through the casting pore, (c) transgranular crack passed through the carbides and (d) crack arrested by carbide.

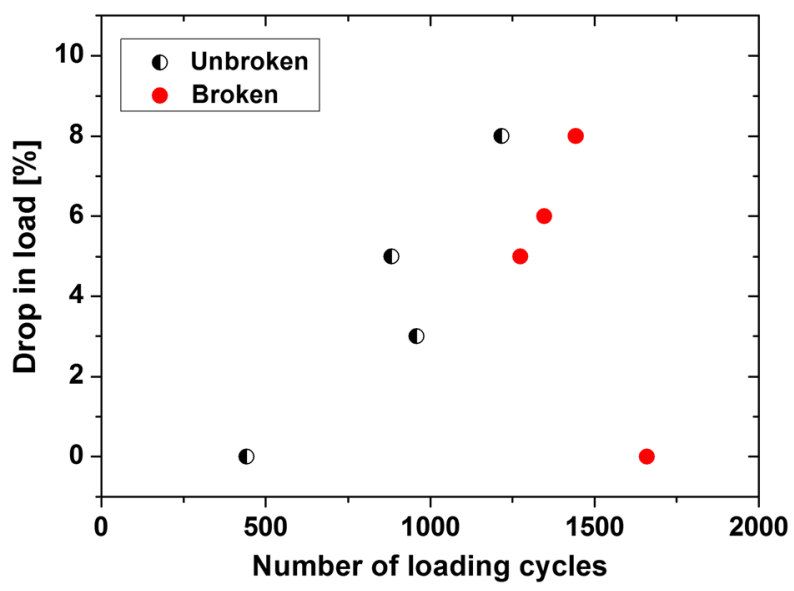

Fig. 12 Fatigue tests interrupted at different load drops to evaluate crack propagation behaviour. the RX layer and propagated into the SX substrate. Similar long cracks can be found in other specimens, for example, a specimen at 959 cycles in Fig. 13c. For the specimen interrupted at 441 cycles, no evident macroscopic cracks were found on the specimen surface. For each of the examined sample, the fatigue crack lengths were measured from the SEM images taken on the longitudinal section. The crack length was normalized by the RX thickness and plotted in terms of the fatigue loading cycles in Fig. 14. It is seen that after 882 loading cycles, the maximal fatigue crack lengths in all samples are larger than RX thickness, suggesting that the primary fatigue crack has penetrated the $\mathrm{RX}$ layer in less than 882 cycles ( $63 \%$ fatigue life).

The SEM observations in the preceding text indicate that the fatigue process of recrystallized SX superalloy
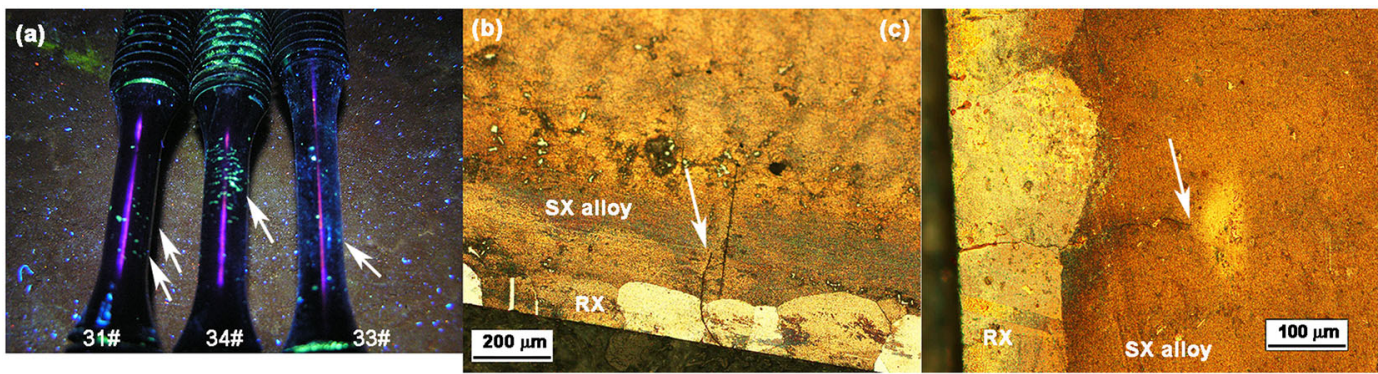

Fig. 13 Fatigue cracking in recrystallization specimens: (a) fluorescence penetrant flaw detection, (b) fatigue crack in sample 31\#, interrupted at 882 cycles and (c) fatigue crack in sample 34\#, interrupted at 959 cycles. 


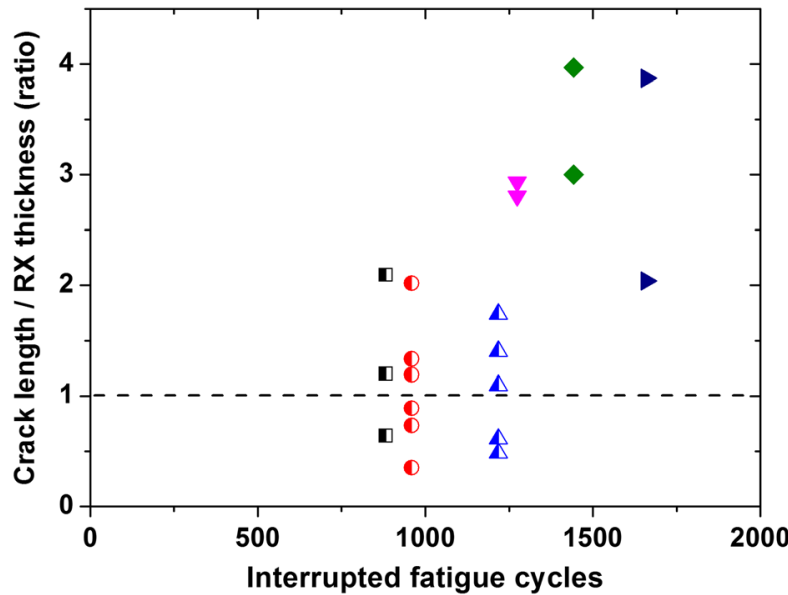

Fig. 14 The crack length in the interrupted fatigue test samples with $150 \mu \mathrm{m}$ recrystallization layer.

is composed of three stages: (i) fatigue crack initiation from casting defects, (ii) short fatigue crack propagation in RX layer and (iii) continued crack propagation in the substrate SX superalloy till failure. Hence, the fatigue life can be given by

$$
N_{f}=N_{i}+N_{p \mathrm{RX}}+N_{f \mathrm{SX}}
$$

Or by two parts if we do not differentiate the life cycles in RX layer,

$$
N_{f}=N_{f \mathrm{RX}}+N_{f \mathrm{SX}}
$$

where $N_{f \mathrm{RX}}$ is the total fatigue life cycles consumed during crack initiation $\left(N_{i}\right)$ and short crack propagation in $\mathrm{RX}$ layer $\left(N_{p \mathrm{RX}}\right), N_{f S \mathrm{SX}}$ is the subsequent crack propagation life in the SX substrate.

The stress intensity factor range $\Delta \mathrm{K}$ when the crack has just passed through the RX layer can be calculated, with a surface ellipse crack assumption, ${ }^{22,23}$ as follows:

$$
\Delta K=\Delta \sigma \sqrt{\pi a} F_{I} / E(k)
$$

where $\Delta \sigma$ is the stress range, $a$ is the crack length or depth, $F_{I}$ is the shape factor, $E(k)$ is the second ellipse integral. ${ }^{22}$

For each of the test samples, the $\Delta \mathrm{K}$ value is calculated using the stress range at mid-life cycle and average RX thickness. In Fig. 15, it is seen that the results fall in the range of 16.6 to $25 \mathrm{MPam}^{1 / 2}$, which exceed the fatigue crack threshold value of about $12 \sim 16 \mathrm{MPam}^{1 / 2}$ for this superalloy. ${ }^{24}$ Generally, it showed a lower $\Delta \mathrm{K}$ value for longer life sample because of a lower applied stress. It is thus indicated that the fatigue crack propagation in the SX substrate is mostly controlled by long crack growth. Hence, the crack growth life after penetrating RX layer $\left(N_{f S X}\right)$ can be estimated using the Paris law, that is,

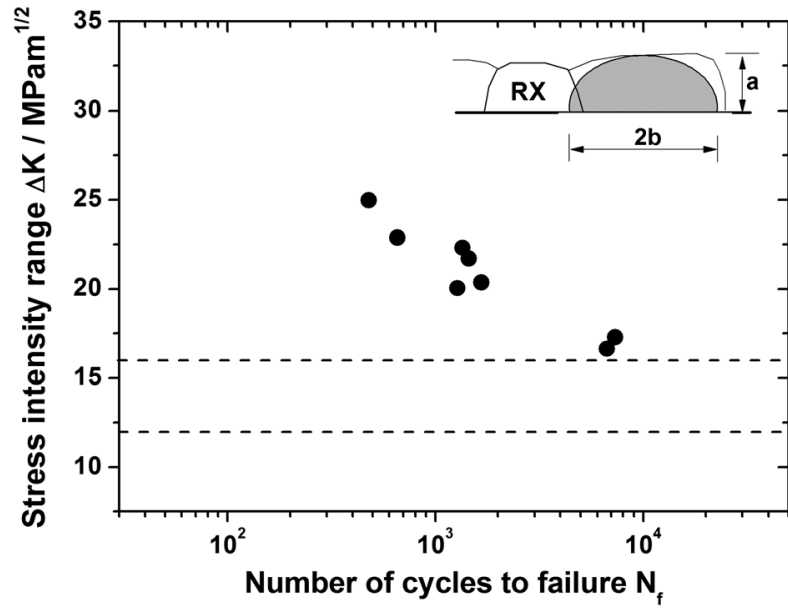

Fig. 15 Stress intensity factor range $\Delta \mathrm{K}$ at the interface between recrystal layer and single crystal substrate.

$$
\frac{d a}{d N}=C(\Delta K)^{n}
$$

where $a$ is the fatigue crack length, $N$ is the fatigue loading cycles, $C$ and $n$ are model parameters.

By integrating the aforementioned formula, the fatigue crack growth life in the SX substrate is given by

$$
N_{f \mathrm{SX}}=\frac{a_{0}^{(1-n / 2)}-a_{f}^{(1-n / 2)}}{C \Delta \sigma^{n} \beta_{1}^{n} \pi^{n / 2}\left(\frac{n}{2}-1\right)}
$$

where $a_{0}$ is the initial crack size, $a_{f}$ is the final crack length, $\beta_{1}$ is the geometry constant, ${ }^{25} \Delta \sigma$ is the applied stress range, $C$ and $n$ are Paris parameters. In this study, $a_{0}$ is taken as the RX layer thickness. $a_{f}$ is set as $2 \mathrm{~mm}$, beyond which the sample is regarded as fractured. The model parameters are taken according to experimental results in literature. ${ }^{4,24}$

For recrystallized samples, the fatigue crack growth life in the SX substrate $N_{f S X}$ is divided by the corresponding total life and plotted in terms of the total life (solid data points in Fig. 16a). It is seen that under large strain amplitude $\varepsilon_{2}$ (with a corresponding fatigue life of 500 cycles), the $N_{p} / N_{f}$ ratio is about $70 \%$, indicating that the RX layer is fractured early in the fatigue life $\left(\sim 30 \% N_{f}\right)$. The $N_{p} / N_{f}$ ratio decreases with increasing fatigue life or decreasing strain amplitude. Under low strain amplitude $\varepsilon_{4}$ (with a corresponding fatigue life of 7000 cycles), an $N_{p} / N_{f}$ ratio of $\sim 23 \%$ is obtained, which indicates that most of fatigue cycles were consumed in the RX layer. For the interrupted fatigue tests performed at strain amplitude of $\varepsilon_{3}$ (solid circular data points in Fig. 16a), the $N_{p} / N_{f}$ ratio is about $40 \%$ (with one data point being $63 \%$ ). These results coincide with the observations in Fig. 13 and quantitative results in Fig. 14. 
(a)

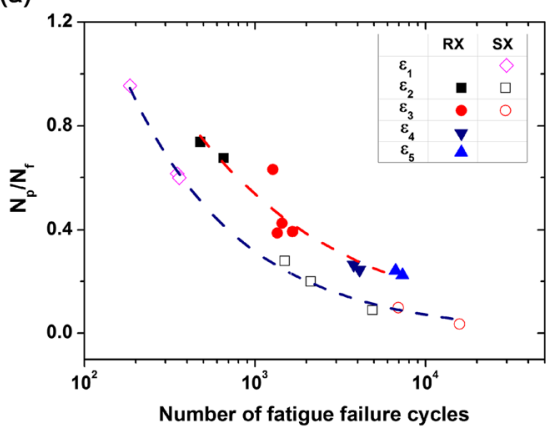

(b)

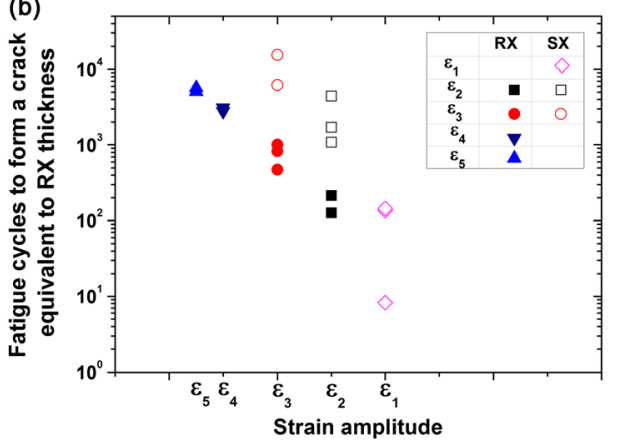

Fig. 16 Analysis based on fatigue crack growth life: (a) life ratio of the fatigue crack growth from recrystallization (RX) layer thickness to final fracture, (b) fatigue cycles required to form a crack equivalent to RX thickness in RX and single crystal samples.

Also plotted is the fatigue crack growth life $N_{p}$ from $a_{0}=150 \mu \mathrm{m}$ to final fracture $\left(a_{f}=2 \mathrm{~mm}\right)$ divided by total fatigue life $N_{f}$ in SX superalloy (denoted by open data points in Fig. 16a), for the sake of comparison. Similar trend is observed that at lower strain amplitude (longer fatigue life), the $N_{p} / N_{f}$ ratio decreases evidently. Also noted is that for raw SX specimens, the $N_{p} / N_{f}$ ratios are much smaller than that in RX samples, indicating that a higher fatigue life percentage was consumed in forming a $150 \mu \mathrm{m}$ crack in SX specimens. Taking the square data points (at strain amplitude $\varepsilon_{2}$ ) in Fig. 16a, for example, the $N_{p} / N_{f}$ ratio of raw SX sample is $\sim 24 \%$, which is about $1 / 3$ of that of RX sample ( $70 \%)$. The relationship between the $N_{p} / N_{f}$ ratio and total fatigue life $\left(N_{f}\right)$ in Fig. 16a can be characterized by a power-law model:

$$
N_{p} / N_{f}=C_{1}\left(N_{f}\right)^{m}
$$

where $C_{1}=27.85, m=-0.65$ for raw SX samples and $C_{1}=14.14, m=-0.47$ for $\mathrm{RX}$ samples.

Moreover, the aforementioned results reflect a fact that the distinct difference in fatigue life mainly occurs in forming a crack equivalent to RX layer thickness. For a better illustration, the fatigue cycles required to form such a crack in both RX samples and SX samples are plotted in Fig. 16b. Note that under strain amplitude of either $\varepsilon_{2}$ or $\varepsilon_{3}$, the number of cycles needed in RX samples is about one order lower than those in SX samples. Hence, the observed remarkable life reduction in Fig. 4 can be rationalized by the early fracture of RX layer in RX samples.

It is known that the surface RX grains tend to show random orientations, compared with the substrate SX alloy in [001] orientation. ${ }^{15}$ This difference in crystal orientation leads to a higher Young's modulus of RX layer, as the modulus in [001] orientation, that is, $E_{[001]}$, is minimum for face-centred cubic crystal. Considering the elasticity theory of crystals, the overall Young's modulus of RX layer can be estimated via Reuss's averaging method, ${ }^{26}$ which is given by

$$
E_{R}=\frac{5}{3 S_{11}+2 S_{12}+S_{44}}
$$

where $S_{11}, S_{12}$ and $S_{44}$ are the three independent compliance constants of SX superalloy. Based on the elastic properties of the studied SX superalloy, ${ }^{4}$ the modulus ratio of RX layer and SX substrate, that is, $E_{R} / E_{[001]}$, is calculated to be 1.55 at room temperature and 1.60 at $550^{\circ} \mathrm{C}$. To check the validity of the aforementioned prediction, the modulus values of RX grains and SX substrate were measured by nano-indentation tests at room temperature. The results indicated average modulus of 168.4 and 118. $6 \mathrm{GPa}$ for RX layer and SX substrate, respectively, yielding a $E_{R} / E_{[001]}$ value of 1.42 , which is close to the predicted result by Reuss's method. The lower experimental value might be caused by less sample data in hard orientations, for example, [111].

In cyclic loading, the RX layer was subjected to a comparable strain level with the SX substrate due to geometry constraint. Hence, the average stress in RX layer is apparently higher than that in SX substrate, which makes it easier to nucleate microcracks in some $\mathrm{RX}$ grains with preferential orientations (e.g. [001]), or containing large casting defects, or both. Also, it rationalizes the occurrence of multiple crack initiations in RX layer as observed earlier. Moreover, this higher stress level would facilitate the subsequent fatigue crack propagation process in RX layer. Regarding the fatigue life reduction by RX grains, RX layer thickness is one of the primary controlling parameters; that is, thicker RX layer would lead to lower fatigue life. This is partly because thicker RX layer means longer cracks formed in the early period of fatigue life. The effect of RX layer thickness was not examined in this study though. Considering that local microstructures, such as grain orientations and misorientations between neighbouring grains, are important in affecting the cyclic plastic deformation and fatigue cracking in recrystallized SX superalloy, crystal plasticity modelling will be used to draw quantitative conclusions in future work. 
Regarding the question whether fatigue fracture is dominated by the cracking process in RX layer, no conclusion has been obtained in open literature except for limited specific cases. Zhang et al. ${ }^{2}$ suggested that the RX layer broke early in the LCF fracture of a turbine blade. Note that intergranular fracture is predominated in their case. In another study of the RX issue in DZ4 DS superalloy, in situ SEM observations proved that more than $90 \%$ of fatigue life was consumed in the RX layer $(80 \sim 300 \mu \mathrm{m})$ under maximum stress of $850 \mathrm{MPa}$ at $R=0.1$. The present study provides evidence that it is crucial to make evaluation for specific strain amplitude or fatigue life, as the life ratio $\left(N_{p} / N_{f}\right)$ follows a decreasing function with fatigue life (Eq. 6). This conclusion is of practical value in evaluating the influence of $\mathrm{RX}$ on the safety of turbine components in terms of LCF failure.

\section{Indication from the fracture of recrystallization}

Review of previous studies on similar superalloys ${ }^{1,3,15}$ indicates that RX microstructure is an important aspect in affecting the strength and the fracture mode of the alien RX grains. With lower annealing temperatures, as used in the work of Bürgel ${ }^{1}$ and Okazaki, ${ }^{3}$ cellular RX with coarser $\gamma / \gamma^{\prime}$ would be formed, which has poor mechanical properties compared with the raw SX. In this study, recalling the RX microstructure in Fig. 3a, the morphology and size of $\gamma^{\prime}$ precipitate are comparable with that of the SX substrate. And there is a high concentration of $\gamma^{\prime}$ precipitate in the RX grain boundary, which may benefit its crack resistance to some extent. Hence, in this study, the main difference between the RX grain and SX substrate is the crystal orientation. The transgranular fracture is mainly induced by crystallographic slipdominated deformation mechanism at this temperature, similar to the fracture mode of DS superalloy ${ }^{15}$ or polycrystalline nickel-based superalloy. ${ }^{23}$ Note that the conclusions obtained here are under the present testing conditions, especially the temperature. At higher temperatures (e.g. $950{ }^{\circ} \mathrm{C}$ ) when intergranular cracking is predominated, multiple cracks along the transverse grain boundaries of RX grains will be expected. In that case, the effect of RX on LCF behaviour should be revisited. Future work will be on quantitative analysis of the orientation and misorientation of the RX grains by electron backscattering diffraction to extract the controlling parameters of fatigue fracture.

\section{CONCLUSION}

By studying the effect of $150 \mu \mathrm{m}$ RX layer on the LCF behaviour of a SX superalloy at $550^{\circ} \mathrm{C}$, the following conclusions have been reached:
1. For raw SX superalloy, casting pores were the primary fatigue crack initiators. For RX specimens, fatigue failure initiated from surface RX layer, more specifically, from the casting pores (primarily), carbides, eutectic or other casting defects. Multiple crack initiation sites were commonly observed in $\mathrm{RX}$ specimens.

2. It is found that RX grains exhibited transgranular cracking, which is distinct from the intergranular fracture mode reported in former studies. It is suggested that the variation of fracture mode of $\mathrm{RX}$ grains is ascribed to the testing conditions, especially the temperature. The results emphasize the practical importance to evaluate the effect of $\mathrm{RX}$ according to the exposed temperature of each turbine component.

3. The fatigue crack growth after penetrating $150 \mu \mathrm{m}$ $\mathrm{RX}$ layer is controlled by long crack growth in this study, based on which the relative importance of the fatigue crack growth life $N_{p}$ (from $\sim 150 \mu \mathrm{m}$ to fracture) has been evaluated. For both RX and SX samples, the life ratio $\left(N_{p} / N_{f}\right)$ follows a decreasing power law with the total life $N_{f}$, suggesting its dependence on the strain amplitude. The results coincide with the observations in the interrupted fatigue tests. $N_{p} /$ $N_{f}$ is much higher in RX sample than that of SX sample under the same strain.

4. The RX layer significantly reduced the fatigue life of SX superalloy. The fatigue cycles to form a crack of $\mathrm{RX}$ thickness were almost an order of magnitude lower than that in raw SX alloy. It is suggested that the earlier crack initiation and promoted crack propagation in RX layer, as well as the trend of multiple crack initiations, can be responsible for the observed fatigue life degradation by RX.

\section{Acknowledgements}

The financial support from Siemens AG Energy and the National Natural Science Foundation of China (Nos. 51071094 and 11372151) is highly acknowledged.

\section{REFERENCES}

1 Bürgel, R., Portella, P. D. and Preuhs J. (2000) Superalloys 2000 (Edited by T. M. Pollock, R. D. Kissinger, R. R. Bowman), TMS, Seven Springs, pp. 229-238.

2 Zhang, W. F., Li, Y. J., Liu, G. Y., Zhao, A. G., Tao, C. H., Tian, J. F. and Yao, G. (2004) Recrystallization and fatigue failure of DS alloy blades. Eng. Failure Anal., 11, 429-437.

3 Okazaki, M., Ohtera, I. and Harada, Y. (2004) Damage repair in CMSX-4 alloy without fatigue life reduction penalty. Metall. Mat. Trans. A, 35, 535-542.

$4 \mathrm{Ma}, \mathrm{X}$. F. (2010) Research on the low cycle fatigue properties of nickel-based single crystal superalloy for large gas turbine. $\mathrm{Ph}$. D. Dissertation, Tsinghua University: Beijing, China. 
5 Cox, D. C., Roebuck, B., Rae, C. M. F. and Reed, R. C. (2003) Recrystallisation of single crystal superalloy CMSX-4. Mater. Sci. Technol., 19, 440-446.

6 Meng, J., Jin, T., Sun, X. F. and Hu, Z. Q. (2010) Effect of surface recrystallization on the creep rupture properties of a nickel-base single crystal superalloy. Mater. Sci. Eng. A 527, 6119-6122.

7 Zhang, B., Liu, C. K., He, Y. H., Tao, C. H. and Lu, X. (2010) Recrystallization of SRR99 single-crystal superalloy: kinetics and microstructural evolution. Rare Metals, 29, 312-316.

8 Zhao, Y., Wang, L., Li, H. Y., Yu, T. and Liu, Y. (2008) Effects of recrystallization on the low cycle fatigue behavior of directionally solidified superalloy DZ40M. Rare Metals, 27, 425-428.

9 Bond, S. D. and Martin, J. W. (1984) Surface recrystallization in a single-crystal nickel-based superalloy. F. Mater. Sci., 19, 3867-3872.

10 Wang, L., Pyczak, F., Zhang, J. and Singer, R. F. (2009) On the role of eutectics during recrystallization in a single crystal nickelbase superalloy - CMSX-4. Int. F. Mater. Res., 100, 1046-1051.

11 Xie, G., Wang, L., Zhang, J. and Lou, L. H. (2008) Influence of recrystallization on the high-temperature properties of a directionally solidified Ni-base superalloy. Metall. Mater. Trans. A, 39A, 206-210.

12 Jo, C. Y. and Kim, H. M. (2003) Effect of recrystallisation on microstructural evolution and mechanical properties of single crystal nickel based superalloy CMSX-2 - part 2 - creep behaviour of surface recrystallised single crystal. Mater. Sci. Technol., 19, 1671-1676.

13 Li, Y. J., Zhang, W. F. and Tao, C. H. (2006) Influence of recrystallized surface layer on high-temperature stress rupture property of DZ4 superalloy. 7. Mech. Strength, 28, 135-137.

14 Shi H. J., Zhang, H. F. and Wu, Y. Q. (2006) Effect of recrystallization on low-cycle fatigue behavior of DZ4 directionally-solidified superalloy. Fract. Strength Solids Vi, Pts 1 and 2, 306-308, 175-180.

$15 \mathrm{Ma}$, X. F. and Shi, H. J. (2013) In situ SEM studies of the low cycle fatigue behavior of DZ4 superalloy at elevated temperature: effect of partial recrystallization. Int 7 Fatigue, doi.org/10.1016/j.ijfatigue.2013.1011.1001.

16 Chung, F. H., Smith, D. K. (1999) Industrial Applications of $\mathrm{X}$-ray Diffraction. CRC Press, New York.

17 Ma, X. F., Shi, H. J., Gu, J. L., Wang, Z. X., Harders, H. and Malow, T. (2008) Temperature effect on low-cycle fatigue behavior of nickel-based single crystalline superalloy. Acta Mech. Solida Sin., 21, 289-297.

18 Shi, H. J., Zhang, H. F. and Wu, Y. Q. (2006) Effect of recrystallization on low-cycle fatigue behavior of DZ4 directionally-solidified superalloy. Key Eng. Mater., 306-308, 175-180.

19 Yi, J. Z., Torbet, C. J., Feng, Q., Pollock, T. M. and Jones, J. W. (2007) Ultrasonic fatigue of a single crystal Ni-base superalloy at $1000^{\circ}$ C. Mater. Sci. Eng. A, 443, 142-149.

$20 \mathrm{Ma}$, X. F., Shi, H. J. and Gu, J. L. (2010) In-situ scanning electron microscopy studies of small fatigue crack growth in recrystallized layer of a directionally solidified superalloy. Mater. Lett., 64, 2080-2083.

$21 \mathrm{Ma}, \mathrm{X}$. F., Gu, J. L. and Shi, H. J. (2014) Low cycle fatigue fracture of a gas turbine Ni-based superalloy with local recrystallizaiton. Mater. Lett., in review.

22 Murakami, Y. (1987) Stress Intensity Factors Handbook. Pergamon press, Oxford.

23 Ma, X. F., Duan, Z., Shi, H. J., Murai, R. and Yanagisawa, E. (2010) Fatigue and fracture behavior of nickel-based superalloy Inconel 718 up to the very high cycle regime. 7. Zhejiang Univ-Sci. A., 11, 727-737.

24 Affeldt, E. E. and Haug, J. (1998) Measurement and results of the crack growth rate in a single crystalline nickel-based superalloy. MTU Aero Engines Munich.

25 Anderson, T. L. (1991) Fracture Mechanics. CRC Press, Boca Raton.

26 den Toonder, J. M. J., van Dommelen, J. A. W. and Baaijens, F. P. T. (1999) The relation between single crystal elasticity and the effective elastic behaviour of polycrystalline materials: theory, measurement and computation. Modell. Simul. Mater. Sci. Eng., 7, 909-928. 